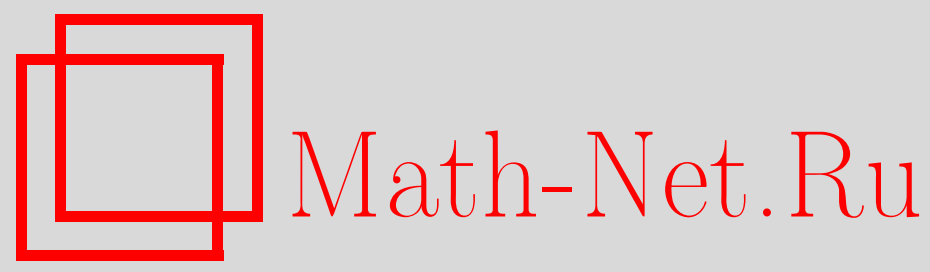

Ю. Л. Павлов, Т. Б. Мюлляри, Предельные распределения числа вершин заданной кратности в лесе случайного отображения с известным числом циклов, Дискрет. матем., 2012, том 24, выпуск 1, 132-139

DOI: https://doi.org/10.4213/dm1177

Использование Общероссийского математического портала Math-Net.Ru подразумевает, что вы прочитали и согласны с пользовательским соглашением http://www . mathnet.ru/rus/agreement

Параметры загрузки:

IP : 54.237 .206 .68

26 апреля 2023 г., $14: 48: 12$ 


\title{
Предельные распределения числа вершин заданной кратности в лесе случайного отображения с известным числом циклов
}

\author{
() 2012 г. Ю. Л. Павлов, Т. Б. Мюлляри
}

\begin{abstract}
Рассматривается случайное однозначное отображение множества объема $n$ в себя, граф которого содержит $m$ циклов. При удалении циклических вершин и дуг оставшийся подграф называется лесом отображения. Для такого леса в случае $n \rightarrow \infty$ и $m=O(\ln n)$ получены предельные распределения числа вершин заданной кратности.
\end{abstract}

Однозначное отображение $S$ конечного множества $T_{n}=\{1,2, \ldots, n\}$ в себя можно представить в виде матрицы

$$
S=\left(\begin{array}{cccc}
1 & 2 & \ldots & n \\
s_{1} & s_{2} & \ldots & s_{n}
\end{array}\right),
$$

где $s_{k}$ обозначает элемент множества $T_{n}$, в который при отображении $S$ переходит элемент $k, k=1,2, \ldots, n$. Такое отображение часто изображается также ориентированным графом с множеством вершин $T_{n}$ и множеством дуг $\left\{\left(1, s_{1}\right),\left(2, s_{2}\right), \ldots,\left(n, s_{n}\right)\right\}$. Число прообразов элемента $k$ при отображении $S$ называется кратностью вершины $k$. Нетрудно видеть, что любая связная компонента графа отображения содержит ровно один цикл. Понятно, что частным случаем такого цикла является петля. Если удалить циклические дуги, то оставшийся подграф представляет собой корневой лес, в котором дуги направлены к циклическим вершинам, являющимся корнями деревьев. Такой подграф будем называть лесом отображения, а число дуг, входящих в любую вершину леса, назовем ее кратностью. Это значит, что для вершин, не являющихся циклическими, понятие кратности вершины отображения совпадает с понятием кратности вершины леса отображения, а у циклических вершин отображения кратность на единицу больше, чем у корней деревьев леса отображения.

Обозначим $\Sigma_{n}$ множество всех однозначных отображений множества $T_{n}$ в себя. Зададим на этом множестве равномерное распределение вероятностей, тогда вероятность каждого отображения равна $n^{-n}$. Такие случайные отображения хорошо известны и подробно изучены. Наиболее полно теория случайных отображений изложена в книге [1].

Обозначим $F_{N, n}$ множество всех ориентированных лесов, состоящих из $N$ корневых деревьев и содержащих $n$ некорневых вершин, все вершины занумерованы. Дуги, как и в лесе отображения, направлены к корням. На множестве $F_{N, n}$ введем равномерное распределение вероятностей.

В [2, 3] были получены предельные распределения максимального объема дерева и числа деревьев заданного объема в случайном лесе из $F_{N, n}$. Эти результаты были исполь- 
зованы при доказательстве предельных теорем для аналогичных характеристик случайного отображения. Идея доказательства этих теорем основана на усреднении результатов о случайных лесах по распределению числа циклических вершин случайного отображения.

В [4] был предложен общий метод доказательства предельных теорем для различных случайных комбинаторных структур, основанный на сингулярном анализе производящих функций. В этой работе были найдены предельные распределения некоторых характеристик случайных отображений. Обозначим

$$
p_{r}=(e r !)^{-1}, \quad \sigma_{r}^{2}=p_{r}\left(1-\left(1+(r-1)^{2} p_{r}\right) .\right.
$$

Обозначим также $v_{r}\left(\Sigma_{n}\right)$ и $\mu_{r}\left(\Sigma_{n}\right)$ число вершин кратности $r$ в случайном отображении и в лесе случайного отображения соответственно. В [4] доказана локальная сходимость распределения случайной величины $\left(v_{r}\left(\Sigma_{n}\right)-n p_{r}\right) /\left(\sigma_{r} \sqrt{n}\right)$ к стандартному нормальному закону при $n \rightarrow \infty$ и любом фиксированном $r$. Аналогичный результат доказан для $\mu_{1}\left(\Sigma_{n}\right)$. Однако для $r \neq 1$ предельные распределения $v_{r}\left(\Sigma_{n}\right)$ и $\mu_{r}\left(\Sigma_{n}\right)$ не совпадают. В этом случае имеет место локальная сходимость распределения $\left(\mu_{r}\left(\Sigma_{n}\right)-n p_{r}\right) /\left(\sigma_{r} \sqrt{n}\right)$ к свертке стандартного нормального закона и распределения Рэлея с параметром $\alpha_{r}=|r-1| p_{r} / \sigma_{r}$. Эти результаты сформулированы ниже в виде теоремы 1. Кроме того, в этой теореме рассматривается также случай $r \rightarrow \infty$. Приведенное далее доказательство теоремы 1 основано, как и в $[2,3]$, на использовании метода усреднения и, следовательно, отличается от доказательства соответствующих утверждений в [4].

Пусть $k$ принимает целые неотрицательные значения. Обозначим

$$
u= \begin{cases}\left(k-n p_{r}\right) /\left(\sigma_{r} \sqrt{n}\right), & \text { если } r=0,1 \text { или } r \rightarrow \infty ; \\ \left(n p_{r}-k\right) /\left(\sigma_{r} \sqrt{n}\right), & \text { если } r \geqslant 2 \text { фиксировано. }\end{cases}
$$

Теорема 1. Пусть $n \rightarrow \infty$. Тогда следующие утверждения справедливы равномерно относительно $k$ таких, что и лежит в любом фиксированном конечном интервале.

(1) Для $r=1$ или $r \rightarrow \infty, n p_{r} \geqslant c>0$, справедлива асимптотическая оценка

$$
\mathbf{P}\left\{\mu_{r}\left(\Sigma_{n}\right)=k\right\}=\frac{1+o(1)}{\sigma_{r} \sqrt{2 \pi n}} \exp \left\{-\frac{u^{2}}{2}\right\} .
$$

(2) При $r \rightarrow \infty, n p_{r} \rightarrow 0$, выполняется соотношение

$$
\mathbf{P}\left\{\mu_{r}\left(\Sigma_{n}\right)=0\right\} \rightarrow 1
$$

(3) Для фиксированных $r \neq 1$ справедлива авсимптотическая оченка

$$
\left.\mathbf{P}\left\{\mu_{r}\left(\Sigma_{n}\right)=k\right\}=\frac{1+o(1)}{\sigma_{r} \alpha_{r}^{2} \sqrt{2 \pi n}} \int_{0}^{\infty} t \exp \left\{-\left((u-t)^{2}+t^{2} / \alpha_{r}^{2}\right) / 2\right)\right\} d t .
$$

Рассмотрим теперь подмножество отображений при условии, что число циклов известно и равно $m$. Обозначим это подмножество $\Sigma_{n, m}$ и введем на нем равномерное распределение вероятностей. Пусть $\mu_{r}\left(\Sigma_{n, m}\right)$ означает число вершин кратности $r$ в лесе такого случайного отображения. Следующая теорема является основным результатом данной статьи.

Теорема 2. Пусть $n \rightarrow \infty$. Тогда следующие утверждения справедливы равномерно относительно $k$ таких, что и лежит в любом фиксированном конечном интервале. 
(1) Для $r=1$ или $r \rightarrow \infty, n p_{r} \geqslant 0, m=o(\ln n)$, справедлива асимптотическая оценка

$$
\mathbf{P}\left\{\mu_{r}\left(\Sigma_{n, m}\right)=k\right\}=\frac{1+o(1)}{\sigma_{r} \sqrt{2 \pi n}} \exp \left\{-\frac{u^{2}}{2}\right\} .
$$

(2) Для $r \rightarrow \infty, n p_{r} \rightarrow 0, m=O(\ln n)$, справедливо соотношение

$$
\mathbf{P}\left\{\mu_{r}\left(\Sigma_{n, m}\right)=0\right\} \rightarrow 1 \text {. }
$$

(3) Для фиксированных $r \neq 1$ u $m=o(\ln n)$ справедлива асимптотическая очеенка

$$
\mathbf{P}\left\{\mu_{r}\left(\Sigma_{n, m}\right)=k\right\}=\frac{1+o(1)}{\sigma_{r} \alpha_{r} \pi \sqrt{n}} \int_{0}^{\infty} \exp \left\{-\frac{1}{2}\left((u-t)^{2}+\frac{t^{2}}{\alpha_{r}^{2}}\right)\right\} d t .
$$

(4) Для фиксированных $r \neq 1 u m / \ln n \rightarrow \gamma, 0<\gamma<\infty$, справедлива асимптотическая оценка

$$
\mathbf{P}\left\{\mu_{r}\left(\Sigma_{n, m}\right)=k\right\}=\frac{2^{\gamma-1} \Gamma(\gamma)(1+o(1))}{\alpha_{r}^{2 \gamma+1} \Gamma(2 \gamma) \sigma_{r} \pi \sqrt{n}} \int_{0}^{\infty} t^{2 \gamma} \exp \left\{-\frac{1}{2}\left((u-t)^{2}+\frac{t^{2}}{\alpha_{r}^{2}}\right)\right\} d t,
$$

где Г $(x)-$ гамма-функичя.

Как видно из теоремы 2, предельные распределения $\mu_{r}\left(\Sigma_{n, m}\right)$ при фиксированных $r \neq 1$ являются свертками стандартного нормального закона и $\chi$-распределения с числом степеней свободы 1 и $2 \gamma+1$ в случаях $m=o(\ln n)$ и $m / \ln n \rightarrow \gamma$ соответственно.

Доказательство теоремь 1. Обозначим $\lambda^{(n)}$ число циклических вершин в графе случайного отображения из $\Sigma_{n}$. Пусть $\mu_{r}\left(F_{N, n}\right)$ означает число вершин кратности $r$ в случайном лесе из $F_{N, n}$. Кратность вершины леса из $F_{N, n}$ имеет тот же смысл, что и в лесе отображения. Нетрудно видеть, что

$$
\mathbf{P}\left\{\mu_{r}\left(\Sigma_{n}\right)=k\right\}=\sum_{N=1}^{n-k r} \mathbf{P}\left\{\lambda^{(n)}=N\right\} \mathbf{P}\left\{\mu_{r}\left(F_{N, n-N}\right)=k\right\} .
$$

Это равенство можно записать в следующем виде:

$$
\sigma_{r} \sqrt{2 \pi n} \mathbf{P}\left\{\mu_{r}\left(\Sigma_{n}\right)=k\right\}=\sum_{i=1}^{4} S_{i},
$$

где

$$
S_{i}=\sum_{N \in M_{i}} \sigma_{r} \sqrt{2 \pi n} \mathbf{P}\left\{\lambda^{(n)}=N\right\} \mathbf{P}\left\{\mu_{r}\left(F_{N, n-N}\right)=k\right\}
$$

и

$$
\begin{array}{ll}
M_{1}=\{N: 1 \leqslant N<\varepsilon \sqrt{n}\}, & M_{2}=\{N: \varepsilon \sqrt{n} \leqslant N \leqslant A \sqrt{n}\}, \\
M_{3}=\left\{N: A \sqrt{n}<N \leqslant n^{2 / 3}\right\}, & M_{4}=\left\{N: n^{2 / 3}<N \leqslant n-k r\right\},
\end{array}
$$

положительные постоянные $\varepsilon$ и $A$ будут определены позднее. Главный вклад в сумму (4) дает слагаемое $S_{2}$. Рассмотрим вероятности $\mathbf{P}\left\{\lambda^{(n)}=N\right\}$ и $\mathbf{P}\left\{\mu_{r}\left(F_{N, n-N}\right)=k\right\}$, входящие в это слагаемое. В [5] доказано, что

$$
\mathbf{P}\left\{\lambda^{(n)}=N\right\}=\frac{N(n-1) !}{n^{N}(n-N) !}
$$


а при $n \rightarrow \infty, z=N / \sqrt{n}$ равномерно по $z \leqslant n^{1 / 6}$

$$
\sqrt{n} \mathbf{P}\left\{\lambda^{(n)}=N\right\}=z e^{-z^{2 / 2}}(1+o(1)) .
$$

Предельное поведение $\mu_{r}\left(F_{N, n}\right)$ рассматривалось в [6]. Из результатов этой статьи следует, что если $N, n \rightarrow \infty, n / N^{2} \rightarrow \beta, 0<\beta<\infty$, то

$$
\mathbf{P}\left\{\mu_{r}\left(F_{N, n}\right)=k\right\}=\frac{1+o(1)}{\sigma_{r} \sqrt{2 \pi n}} e^{-v^{2} / 2}
$$

равномерно относительно целых неотрицательных $k$, таких, что

$$
v=\left(k-(N+n) p_{r}+(r-1) p_{r} \sqrt{(N+n) / \beta}\right) /\left(\sigma_{r} \sqrt{N+n}\right)
$$

лежит в любом фиксированном конечном интервале; при этом (7) остается справедливым и при $r \rightarrow \infty$.

Рассмотрим сначала случай $r=1$. Из (2) и (7) следует, что

$$
S_{2}=e^{-u^{2} / 2}(1+o(1)) \sum_{M_{2}} \mathbf{P}\left\{\lambda^{(n)}=N\right\} .
$$

Поскольку $\varepsilon$ мы можем выбрать сколь угодно малым, а $A$ сколь угодно большим, из (6) и (8) находим, что

$$
S_{2}=e^{-u^{2} / 2}(1+o(1))
$$

Легко видеть также, что (9) остается справедливым и при $r \rightarrow \infty$.

Пусть теперь $r=0$. Из (6) и (7) получаем, что

$$
S_{2}=(1+o(1)) \sum_{M_{2}} \exp \left\{-\frac{\left(k-n p_{0}-N p_{0}\right)^{2}}{2 \sigma_{0}^{2} n}\right\} \frac{N}{n} e^{-N^{2} / 2 n} .
$$

Отсюда и из (2), вводя обозначение $y=N / \sqrt{n}$, выводим, что

$$
S_{2}=(1+o(1)) \sum_{y} \exp \left\{-\frac{\left(u \sigma_{0}-p_{0} y\right)^{2}}{2 \sigma_{0}^{2}}\right\} y e^{-y^{2} / 2} \frac{1}{\sqrt{n}},
$$

где суммирование по $y$ проводится от $\varepsilon$ до $A$ с шагом $1 / \sqrt{n}$. Поскольку в правой части этого равенства находится интегральная сумма, выбирая достаточно малое $\varepsilon$ и достаточно большое $A$, и полагая $t=p_{0} y / \sigma_{0}$ и $r=0$, получаем, что

$$
S_{2}=\frac{1+o(1))}{\alpha_{r}^{2}} \int_{0}^{\infty} t \exp \left\{-(u-t)^{2}+\frac{t^{2}}{\alpha_{r}^{2}}\right\} d t .
$$

Нетрудно проверить, что равенство (10) справедливо и для $r \geqslant 2$. В этом случае

$$
k=n p_{r}-u \sigma_{r} \sqrt{n}
$$

и используется подстановка

$$
t=\frac{(r-1) p_{r} y}{\sigma_{r}}
$$


Из (4), (9) (10) следует, что для доказательства утверждений 1 и 3 достаточно показать, что

$$
S_{i}=o(1), \quad i=1,3,4 \text {. }
$$

Введем вспомогательные независимые случайные величины $\xi_{1}, \xi_{2}, \ldots$ с общим распределением Пуассона:

$$
p_{k}(\lambda)=\mathbf{P}\left\{\xi_{1}=k\right\}=\frac{\lambda^{k} e^{-\lambda}}{k !}, \quad k=0,1, \ldots, \quad \lambda>0,
$$

а также случайные величины $\xi_{1}^{(r)}, \xi_{2}^{(r)}, \ldots$, для которых

$$
\mathbf{P}\left\{\xi_{i}^{(r)}=k\right\}=\mathbf{P}\left\{\xi_{i}=k \mid \xi_{i} \neq r\right\}, \quad i=1,2, \ldots
$$

Обозначим

$$
\zeta_{N}=\xi_{1}+\ldots+\xi_{N}, \quad \zeta_{N}^{(r)}=\xi_{1}^{(r)}+\ldots+\xi_{N}^{(r)}
$$

С помощью обобщенной схемы размещения частиц по ячейкам [1], в [6] установлено, что для всех $\lambda>0$ справедливо равенство

$$
\mathbf{P}\left\{\mu_{r}\left(F_{N, n}\right)=k\right\}=\left(\begin{array}{c}
N+n \\
k
\end{array}\right) p_{r}^{k}(\lambda)\left(1-p_{r}(\lambda)\right)^{N+n-k} \frac{\mathbf{P}\left\{\zeta_{N+n-k}^{(r)}=n-k r\right\}}{\mathbf{P}\left\{\zeta_{N+n}=n\right\}} .
$$

Мы будем использовать два значения параметра $\lambda$. Если $N, n \rightarrow \infty$ так, что $n / N^{2} \rightarrow 0$, то $\lambda=n /(N+n)$, а в других случаях $\lambda=1$. В [7] показано, что если $N=o(n)$, то

$$
\frac{\mathbf{P}\left\{\zeta_{n-k}^{(r)}=n-N-k r\right\}}{\mathbf{P}\left\{\zeta_{n}=n-N\right\}} \leqslant C_{1}
$$

здесь и далее символы $C_{1}, C_{2}, \ldots$ означают некоторые положительные постоянные. Из (5) следует, что

$$
\mathbf{P}\left\{\lambda^{(n)}=N\right\}<N / n,
$$

поэтому из (1), (4), (12) и (13) находим, что при достаточно малом $\varepsilon$

$$
S_{1}<C_{2} \varepsilon^{2},
$$

следовательно, равенство (11) справедливо для $i=1$.

Рассмотрим следующую сумму $S_{3}$. Из (1), (4), (6), (13), (14) и нормального приближения биномиального распределения получаем, что

$$
S_{3}<C_{3} \mathbf{P}\left\{\lambda^{(n)}>A \sqrt{n}\right\} \rightarrow 0 .
$$

Из (5) легко получить, что для $N \in M_{4}$ справедливо неравенство

$$
\mathbf{P}\left\{\lambda^{(n)}=N+1\right\} \leqslant \mathbf{P}\left\{\lambda^{(n)}=N\right\},
$$

поэтому из (4) и (6) находим, что

$$
S_{4}<C_{4} n^{7 / 6} e^{-n^{1 / 3} / 2} \rightarrow 0 .
$$


Таким образом, все соотношения (11) установлены, поэтому утверждения 1 и 3 верны, и для завершения доказательства теоремы 1 осталось рассмотреть утверждение 2.

Из (3) и (6) следует, что

$$
\mathbf{P}\left\{\mu_{r}\left(\Sigma_{n}\right)=k\right\}=\sum_{\varepsilon \sqrt{n} \leqslant N \leqslant A \sqrt{n}} \mathbf{P}\left\{\lambda^{(n)}=N\right\} \mathbf{P}\left\{\mu_{r}\left(F_{N, n-N}\right)=k\right\}+\delta,
$$

где величина $\delta$ может быть сделана сколь угодно малой выбором достаточно больших $n$ и $A$ и достаточно малого $\varepsilon$.

Обозначим через $\eta$ максимальное число частиц в ячейке при равновероятном размещении $n$ разных частиц по $N+n$ помеченным ячейкам. В [8] показано, что если $N, n \rightarrow \infty$ так, что $n / N^{2} \rightarrow \gamma, 0<\gamma<\infty$, и $r=r(\gamma, N, n)$ выбрано так, что $n p_{r}(n /(N+n)) \rightarrow \beta$, $0<\beta<\infty$, то

$$
\mathbf{P}\{\eta=r+1\} \rightarrow e^{-\beta}, \quad \mathbf{P}\{\eta=r\} \rightarrow 1-e^{-\beta} .
$$

Из результатов книги [1] следует, что распределение числа вершин кратности $r$ в случайном дереве из $F_{1, n}$ совпадает с распределением числа ячеек в классической схеме размещения $n$ частиц по $n+1$ ячейке. Аналогичная ситуация имеет место и для леса из $F_{N, n}$, если число ячеек равно $N+n$ (см. $\left.[5,6]\right)$. Поэтому из (1) и (12) вытекает, что если $r$ выбрано так, что $n p_{r} \rightarrow 0$, то и $n p_{r}(n /(N+n)) \rightarrow 0$, и из (20) находим, что

$$
\mathbf{P}\left\{\mu_{r}\left(F_{N, n-N}\right)=0\right\} \rightarrow 1 \text {. }
$$

Отсюда и из (19) получаем второе утверждение теоремы 1.

Доказательство теоремы 2. По аналогии с (4) запишем равенство:

$$
\sigma_{r} \sqrt{2 \pi n} \mathbf{P}\left\{\mu_{r}\left(\Sigma_{n, m}\right)=k\right\}=\sum_{i=1}^{4} S_{i}
$$

где

$$
S_{i}=\sum_{N \in M_{i}} \sigma_{r} \sqrt{2 \pi n} \mathbf{P}\left\{\lambda^{(n, m)}=N\right\} \mathbf{P}\left\{\mu_{r}\left(F_{N, n-N}\right)=k\right\},
$$

$\lambda^{(n, m)}-$ число циклических вершин в графе случайного отображения из $\Sigma_{n, m}$,

$$
M_{1}=\{N: m \leqslant N<\varepsilon \sqrt{n}\},
$$

а области $M_{2}-M_{4}$ определены так же, как в (4). Главный вклад в сумму (21) дает слагаемое $S_{2}$.

Рассмотрим сначала случай $r=1$. В [9] показано, что при $n \rightarrow \infty, m / \ln n \rightarrow 0$ равномерно по $z=N / \sqrt{n}$ в любом фиксированном конечном интервале

$$
\sqrt{n} \mathbf{P}\left\{\frac{\lambda^{(n, m)}}{\sqrt{n}}=z\right\}=\sqrt{\frac{2}{\pi}} e^{-z^{2} / 2}(1+o(1)),
$$

а если $m / \ln n \rightarrow \gamma$, где $\gamma-$ некоторая положительная постоянная, то для таких $z$

$$
\sqrt{n} \mathbf{P}\left\{\frac{\lambda^{(n, m)}}{\sqrt{n}}=z\right\}=\frac{2^{\gamma} \Gamma(\gamma)}{\sqrt{2 \pi \Gamma(2 \gamma)}} z^{2 \gamma} e^{-z^{2} / 2}(1+o(1)) .
$$


Из (2), (7) и (21)-(23) нетрудно получить, как и в (8), что

$$
S_{2}=e^{-u^{2} / 2}(1+o(1)) .
$$

Ясно также, что (24) сохраняет силу и при $r \rightarrow \infty$.

Пусть теперь $m=o(\ln n), r=0$. Используя (7), (21) и (22), находим, что

$$
S_{2}=(1+o(1)) \sqrt{\frac{2}{\pi}} \sum_{M_{2}} \exp \left\{-\frac{\left(k-n p_{0}-N p_{0}\right)^{2}}{2 \sigma_{0}^{2} n}\right\} \exp \left\{-\frac{N^{2}}{2 n}\right\} \frac{1}{\sqrt{n}},
$$

откуда, как и при доказательстве (10), получаем, что при $r=0$

$$
S_{2}=\frac{1+o(1))}{\alpha_{r}} \sqrt{\frac{2}{\pi}} \int_{0}^{\infty} \exp \left\{-\frac{1}{2}\left(\frac{t^{2}}{\alpha_{r}^{2}}+(u-t)^{2}\right)\right\} d t .
$$

Нетрудно видеть также, что (25) остается в силе и при $r \rightarrow \infty$.

Теперь нам нужно установить, что

$$
S_{i}=o(1), \quad i=1,3,4 .
$$

В [9] показано, что

$$
\mathbf{P}\left\{\lambda^{(n, m)}=N\right\}=\frac{\mathbf{P}\left\{\lambda^{(n)}=N\right\} \mathbf{P}\left\{\varkappa_{N}=m\right\}}{\mathbf{P}\left\{\xi^{(n)}=m\right\}},
$$

где $\varkappa_{N}$ - число циклов в случайной подстановке степени $N$ с равномерным распределением на множестве всех подстановок, а $\xi^{(n)}$ - число циклов в случайном отображении из $\Sigma_{n}$. Из лемм 2 и 10 в [9] следует, что если $N \rightarrow \infty$ и $m=o(\ln N)$, то

$$
\mathbf{P}\left\{\varkappa_{N}=m\right\}=\frac{(\ln N)^{m-1}}{N(m-1) !}(1+o(1)),
$$

а если $n \rightarrow \infty$ и $m=o(\ln N)$, то

$$
\mathbf{P}\left\{\xi^{(n)}=m\right\}=\sqrt{\frac{\pi}{2}} \frac{(\ln \sqrt{n})^{m-1}}{\sqrt{n}(m-1) !}(1+o(1)) .
$$

Тогда из (13)-(15), (21), (27)-(29) и нормального приближения биномиального распределения находим, как и при оценке (16), что величину $S_{1}$ можно сделать сколь угодно малой выбором достаточно малого $\varepsilon$. Аналогично (17) получаем, что

$$
S_{3}<C_{5} \mathbf{P}\left\{\lambda^{(n, m)}>A \sqrt{n}\right\},
$$

и для доказательства (26) при $i=3$ остается применить (22) и учесть возможность выбора сколь угодно большого $A$.

Пусть $N \in M_{4}$. Тогда из (27)-(29) следует, что

$$
\mathbf{P}\left\{\lambda^{(n, m)}=N\right\} \leqslant C_{6} \mathbf{P}\left\{\lambda^{(n)}=N\right\} e^{m} n^{-1 / 6} .
$$

Отсюда находим, как и при доказательстве (18), что

$$
S_{4}<C_{7} n \exp \left\{m-n^{1 / 3} / 2\right\},
$$


и $S_{4} \rightarrow 0$ в силу условия $m=o(\ln n)$. Таким образом, (26) выполняется и в этом случае, поэтому из (24)-(26) выводим утверждения 1 и 3.

Пусть $m=\gamma \ln n+o(\ln n)$. Рассмотрим $S_{2}$. Для $r=0$ из (7) и (23) получаем, что

$$
S_{2}=\frac{2^{\gamma} \Gamma(\gamma)(1+o(1))}{\sqrt{2 \pi} \Gamma(2 \gamma)} \sum_{M_{2}} \exp \left\{-\frac{\left(k-n p_{0}-N p_{0}\right)^{2}}{2 \sigma_{0}^{2} n}\right\} \exp \left\{-\frac{N^{2}}{2 n}\right\}\left(\frac{N}{\sqrt{n}}\right)^{2 \gamma} \frac{1}{\sqrt{n}} .
$$

Аналогично доказательству (25) находим отсюда, что при $r=0$

$$
S_{2}=\frac{2^{\gamma} \Gamma(\gamma)(1+o(1))}{\sqrt{2 \pi} \Gamma(2 \gamma) \alpha_{r}^{2 \gamma+1}} \int_{0}^{\infty} t^{2 \gamma} \exp \left\{-\frac{1}{2}\left((u-t)^{2}+\frac{t^{2}}{\alpha_{r}^{2}}\right)\right\} d t .
$$

Согласно лемме 8 в [9], в рассматриваемом случае

$$
\mathbf{P}\left\{\varkappa_{N}=m\right\}=\frac{(\ln N)^{m-1}}{(m-1) ! N \gamma \Gamma(\gamma)}(1+o(1)),
$$

поэтому справедливость соотношения (26) при $i=1$ нетрудно установить, как и ранее, используя (13)-(15), (27) и равенство

$$
\mathbf{P}\left\{\xi^{(n)}=m\right\}=\sqrt{\frac{\pi}{2}} \frac{(\ln \sqrt{n})^{m-1}}{\sqrt{n}(m-1) ! 2^{\gamma} \Gamma(\gamma)}(1+o(1)),
$$

доказанное в [9]. Для $S_{3}$ оценка (30) верна в силу (23) и нормального приближения биномиального распределения. Оценки вида (31) и (32) нетрудно получить из (27), (34) и (35). Таким образом, утверждение 4 теоремы 2 справедливо в силу (21), (26) и (33).

Утверждение 2 теоремы 2 доказывается аналогично утверждению 2 теоремы 1 , при этом используются соотношения (22) и (23).

\section{Список литературы}

1. Колчин В. Ф., Случайные отображения. Наука, Москва, 1984.

2. Павлов Ю. Л., Асимптотическое распределение максимального объема дерева в случайном лесе. Теория вероятностей и ее применения (1977) 22, 523-533.

3. Павлов Ю. Л., Предельные теоремы для числа деревьев заданного объема в случайном лесе. Матем. сб. (1977) 103, 392-403.

4. Drmota M., Soria M., Images and preimages in a random mapping. 1045-1062 (1997) 31,

5. Harris B., Probability distributions related to random mappings. Ann. Math. Statist. (1960) 10, 246-269.

6. Mylläri T., Pavlov Yu., Limit distribution of the number of vertices of a given outdegree in a random forest. J. Math. Sci. (2006) 138, 5424-5433.

7. Mylläri T., Studies in the theory of random forests. Åbo Akademi University, Åbo, 2004.

8. Колчин В. Ф., Севастьянов Б. А., Чистяков В. П., Случайные размещения. Наука, Москва, 1976.

9. Павлов Ю. Л., О случайных отображениях с ограничениями на число циклов. Труды МИАН СССР (1986) 177, 122-132. 